\title{
Impact of Intrinsic Factors and COVID-19 Pandemic on the Use of Technology
}

\author{
Ala Sarah Alaqra* \\ Karlstad University \\ Karlstad, Sweden \\ as.alaqra@kau.se
}

\author{
Agnieszka Kitkowska* \\ Karlstad University \\ Karlstad, Sweden \\ agnieszka.kitkowska@kau.se
}

\begin{abstract}
The unprecedented pandemic of the infectious coronavirus disease (COVID-19) is still ongoing. Considering the limitations and restrictions imposed by COVID-19, we explored the role of technology and the extent of usage by end-users. In our online survey, we investigated users' perspectives on their use of technologies in different contexts (e.g., work, entertainment), taking into consideration intrinsic factors such as health consciousness, perceived social isolation, and pandemic-related concerns. Results from 218 respondents show a significant increase in technology use in all investigated contexts after the pandemic occurred. Moreover, the results suggest that different factors may contribute to such increases, depending on the context. It appears that perceived social isolation, concerns about the pandemic, and tracking have the most prominent influence on different use of technology. Furthermore, open-ended responses include beneficial opportunities, concerns \& consequences, and behavioral transformations \& adaptations due to COVID-19. Our findings provide insights for designing and developing new technologies, especially for communication and entertainment, to support users' needs during a pandemic.
\end{abstract}

\section{CCS CONCEPTS}

- Human-centered computing $\rightarrow$ Empirical studies in $\mathrm{HCI}$.

\section{KEYWORDS}

COVID-19 pandemic, technology use, intrinsic factors, behavior, attitude

\section{ACM Reference Format:}

Ala Sarah Alaqra and Agnieszka Kitkowska. 2021. Impact of Intrinsic Factors and COVID-19 Pandemic on the Use of Technology. In CHI Conference on Human Factors in Computing Systems Extended Abstracts (CHI '21 Extended Abstracts), May 8-13, 2021, Yokohama, Japan. ACM, New York, NY, USA, 7 pages. https://doi.org/10.1145/3411763.3451669

\section{INTRODUCTION}

The current pandemic of the coronavirus, or COVID-19 [45], has taken the world by surprise. The disease has already exceeded the

${ }^{*}$ Both authors contributed equally to this research.

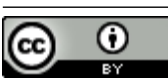

This work is licensed under a Creative Commons Attribution International 4.0 License.

CHI '21 Extended Abstracts, May 8-13, 2021, Yokohama, Japan

(c) 2021 Copyright held by the owner/author(s).

ACM ISBN 978-1-4503-8095-9/21/05.

https://doi.org/10.1145/3411763.3451669
83 million reported infections and over 1.8 million deaths globally as of the 3rd of January, 2021 [43, 46, 47]. Different regulations and guidelines have been implemented early on to limit the infection and spread in many countries. Tighter restrictions include army and police interference, such as the case in Romania, Armenia, and Moldova [33], whereas mere social distancing has been advised in Sweden [32]. The impact of such a pandemic is affecting not only healthcare and economies, but also people's lifestyles. Technology, Information and Communications Technology (ICT) specifically, plays a significant role in facilitating services and mitigating hindrances imposed by restrictions. Our study aims to investigate people's perspectives on the role of technology during the coronavirus pandemic. We empirically investigate whether the restrictions affect the technology use-increase or decrease in the different contexts (e.g., work, communication). We also examine select individual factors that influence technology use changes to understand how technology might help people maintain their well-being throughout similar crises. Human-Computer Interaction (HCI) researchers and designers could use such knowledge to customize and improve the current technological solutions, fulfilling people's needs related to the pandemic.

Considering the limited knowledge about people's perception of technology in the time of the global pandemic, in our research, we aim to answer the following questions:

- What are the effects of COVID-19 on technology usage by participants?

- What intrinsic factors (health consciousness, perceived isolation, concerns about tracking and pandemic) contribute to changes in the use of technology in different contexts?

\section{BACKGROUND}

\subsection{Health and Impact of Technology}

With the constant rise of information online, the availability of technologies and portals providing access to them correspondingly increases. We live in an era where users are consuming online content for various purposes. According to the Health Information National Trends Survey (HINTS) in 2007, $80.2 \%$ of users in the United States used the internet for seeking health information [28]. Studies show that such information seeking is a growing trend among users $[4,11,29]$.

During COVID-19, people have been seeking information about the pandemic and the disease, and have been acting upon it rapidly [2]. It is thought that people who are more health-conscious tend to seek more health information online than those who engage in less healthy lifestyles [10]. Health consciousness refers to the awareness of a person concerned with their health and the actions 
they would take accordingly in their lifestyle and diet $[1,19]$. Past research suggests that health-consciousness might relate to the use of digital technology. Particularly, it was shown to influence the extent of using health-apps, thus positively impacting the use of such applications by people who are health concerned [7]. Our study investigates health-consciousness, considering its potential influence on the use of technology to seek information or healthtracking, which both might have become critical for many people due to the pandemic.

Due to the restrictions imposed by COVID-19, overall physical activities and encounters are reduced, which could be resulting in health concerns. Health is defined by the World Health Organization (WHO) as the "state of complete physical, mental and social well-being and not merely the absence of disease or infirmity" [44]. Hence, not only is being infected with the COVID-19 disease a risk itself, but indirect consequences of the pandemic may pose threats to individual's health. People everywhere are limiting their social interactions with others, working from home, and avoiding unnecessary gatherings at the very least. Such limitations to current lifestyles alongside the reduced physical activities and interactions do have consequences to the overall well-being, i.e., health. Several studies have emerged providing health information containing recommendations to help mitigate health consequences physiologically (e.g., obesity and cardiovascular diseases), physically (e.g., inactivity), and mentally (e.g., home confinement stress) $[6,23,27,31,48]$. Technology plays a vital role in communicating information, facilitating tasks remotely, and providing possibilities to cope with the physical restrictions imposed by COVID- 19 . In fact, it is not only a coping mechanism to expand health services and communication, but also an opportunity to expand flexibility to users beyond the current pandemic [14].

\subsection{Technology Use Contexts During COVID-19}

The use of technology is growing in popularity in different application areas and contexts. For example, in the work context, technology has played an important role in enabling remote working (or working from home), especially with COVID-19 it became a significant phenomenon [3, 21]. In education and information acquisition, technology facilitates the dissemination of knowledge, which supports the availability of course materials and distance learning. During COVID-19, the adoption of distance learning has been shown to be necessary $[9,24,36]$. In addition, the emergence of emergency distance teaching and learning has been shown to happen around the world [24]. Commonly, video games, YouTube, and streaming services such as Netflix, are examples of mediums providing entertainment and leisure for consumers. Increased use of such services for entertainment and leisure has been noted as one way to cope with conditions during COVID-19 [22, 30]. As for Health, fitness applications and wearable devices have been increasing for both medical and personal usage [16]. Many health mobile applications relating to COVID-19 have been emerging since the beginning of the pandemic in support of users' well-being $[26,48]$.

Technology has opened the portal for many means to connect with people and to socialize online. Several platforms exist for networking, sharing interests, and communicating with close acquaintances and strangers alike. Connectivity became more important during COVID-19 and such technologies became crucial in different application areas such as video conferencing, entertainment, social media chats, and health [42].

2.2.1 Tracking and COVID-19. On the dashboard of WHO web page, the dashboard of WHO corona virus disease, information about COVID-19 and the tracking of the spread of the disease around the world is available [43]. At a more granular level, there are applications for tracking individual symptoms of the disease as well as governmental tracking across many countries [40]. Tactics for tracking the disease vary from self-report applications, infodemiology approaches, and surveillance tools. Self-reported applications, such as the symptom tracker of the disease, have shown data that aids in prediction [49]. An Infodemiology approach has been followed during COVID-19 for predicting and tracking the pandemic [25]. Infodemiology is "the science of distribution and determinants of information in an electronic medium, specifically the Internet, or in a population, with the ultimate aim to inform public health and public policy" [12]. Apart from the benefits perceived by those tracking applications, privacy could be threatened due to those applications. Consequently, ethical considerations for designing COVID-19 tracking applications are recommended [20], for instance, through implementing principles defined in a framework of contextual integrity enabling greater privacy [41]. Since tracking is not in all cases voluntary, ethical considerations are important, as well as user's perspectives on tracking applications. In our survey, we included questions inquiring about user's acceptance of opting into a tracking service and/or if they are interested in tracking information.

2.2.2 Social Effects and Isolation. One of the effects of the COVID19 pandemic and lock-downs enforced on many societies is increasing social isolation. Typically, social isolation is associated with many different factors present in individuals' lives, such as solitary habitation, no formal romantic relationships, and lack of social networks and interactions [8]. Furthermore, social isolation is often considered a problem of the elderly because the elderly are more likely to experience 'physical' solitude [14]. However, in times of crisis, such as a global pandemic, people of all ages might become social isolation victims. A component of social isolation is perceived isolation "characterized by the subjective experience of a shortfall in one's social resources such as companionship and support” [8], including loneliness and social support. Perceived isolation might have strong connotations with both physical (e.g., cardiovascular disease, inflammation) and mental health (e.g., depression) [8, 14]. Loneliness is a serious problem affecting people's health even in the pre-COVID-19 era. For instance, in Europe, the USA, and China, both loneliness and social isolation have had adverse effects on overall-well being [18]. Recent reports on the effects of the COVID19 pandemic show that the virus has substantial sociopsychological impacts, including effects of isolation that may lead to serious mental health illnesses [35]. Usually, different disasters and pandemics bring such adverse effects, which are exacerbated by a lack of social support and loneliness [35].

Technology might be used to reduce the negative effects of increasing isolation, and WHO emphasizes the importance of maintaining social networks and connections with others through digital means [37]. Past work showed that the use of technologies 
such as video conferencing could help with overcoming depression and loneliness [14]. However, some studies suggest adverse effects of technology use on mental well-being [14]. For instance, social media might have a beneficial impact on mental health, but there is an overwhelming evidence that they might lead to harmful behaviors, particularly among youths [17]. Moreover, early COVID19 research revealed that the use of more traditional media (e.g., TV, radio) might benefit psychological well-being, but the modern media (e.g., online news, social media) may have adverse effects: increasing depression, stress, and anxiety [5]. In this research, we investigate perceived isolation to identify whether people might use information technology to mitigate the effects of social isolation.

\section{METHOD}

We designed an online survey (see Appendix for details) to answer our research questions. To participate, each respondent was required to acknowledge an informed consent form, ensuring the study's full understanding, purpose, and data collection and processing details. The survey contained questions about technology use-pre- (5 questions) and during-pandemic (5 questions)-for work, gathering information/education, entertainment, health, and communication. Next, we measured perceived isolation (loneliness and social support) and health consciousness with scales validated in past research $[8,19]$. We also incorporated questions related to attitudes and concerns about the pandemic, including an openended question about COVID-19 and its impact on technology use and potential self-reported behavioral changes. Lastly, we collected basic demographics and information related to experiences with the pandemic. We implemented the survey with the SoSci survey tool, and collected data in summer 2020. The average completion time was 4.4 minutes.

\subsection{Ethical considerations}

Our survey has been granted an ethical approval by the university's ethical advisor according to the national ethics review act. The survey was designed so that no personally identifying information is collected, in addition to anonymizing the survey. We provided information using our consent forms to the survey participants. In addition, the tool is installed on the University's servers, and only responsible researchers can access the data.

\subsection{Participants}

We gathered participants through a snowball-sampling method, mostly through social media and with the use of other freely available channels (e.g., SamplSize subreddit). Participation in the survey was entirely voluntary; everyone above 18 years old could participate. Overall we collected 219 responses and removed one because of the uniformity of the responses to all questions. More than half of the participants, $N=129$ identified themselves as females, $N=82$ as males, $N=5$ selected 'Other' and $N=2$ preferred not to say. Most of participants were young; $N=45$ 18-28 yo, $N=7029$-38 yo, $N=3639-48$ yo, $N=3849-58$ yo, and the remaining $N=29$ being above 59 yo. The largest cohorts in our sample were from Ireland $(N=47)$, Sweden $(N=40)$, USA $(N=34)$, UK $(N=21)$, and Brazil $(N=20)$. Our participants had diverse professional background, from fields such as information technology $(N=36)$, teaching and education $(N=24)$, healthcare $(N=21)$, business, consulting and management $(N=14) . N=21$ participants were students, and $N=19$ unemployed.

We asked participants about their attitudes toward tracking application because of this study's general interest in the COVID-19 pandemic. Only 12 participants indicated that they used the tracking application before. We asked participants whether they would like to use a tracking application, and 116 answered 'Yes,' leaving $42.7 \%$ not willing to be tracked.

\section{RESULTS}

To ensure the validity of latent constructs measured in the study, we ran Principal Component Analysis (PCA) when appropriate. We used PCA to check whether the utilized scales match the original maximum number and nature of factors [38]. If PCA resulted in significant inconsistencies with the original numbers of factors, we would perform exploratory factor analysis, as recommended in [38]. We also checked scales' reliability with the Cronbach $\alpha$ (commonly recommended score is above .7 [15]).

Health consciousness. This construct was measured with a validated instrument acquired from [19] consisting of five items. The results of PCA were satisfactory, with Kaiser-Mayer Olkin (KMO) score .72 and a significant Bartlett test for sphericity. All items loaded into one factor, explaining $45 \%$ of variance. We checked the scale's reliability, which was not perfect, with Cronbach $\alpha=.68$. Regardless, we kept the original scale because removing items did not increase reliability. We used the mean score to compute the health consciousness variable.

Perceived isolation. We measured two dimensions of perceived isolation: loneliness and social support using scale acquired from [8]. However, we treated these as measurements of separate constructs because we modified items measuring social support. Loneliness was assessed with three items. Principal Axis Factoring (PAF) resulted in acceptable KMO score .73 and significant Bartlett test. All items loaded into one factor, explaining $80 \%$ of variance. The Cronbach $\alpha=$.87. To compute the loneliness variable we used scores' mean. The social support dimension was measured with six items. Because the questions concerned reliance on family, friends, or spouses, we modified the scale and offered participants to select the option: 'I have none.' We ran PAF, and the KMO score was .71, and Bartlett test significant. Although the PAF items did not load into one factor, we kept the original scale because it resulted in the highest reliability, Cronbach $\alpha=.79$. To compute the social support variable, we used scores' means.

Pandemic tracking. We asked participants about their attitudes towards tracking and sharing information about the pandemic. We ran PCA, and the KMO was lower than expected, .65, and Bartlett's test was significant. All items loaded highly in one factor, explaining $65 \%$ of variance. The reliability of the scale was good, with Cronbach $\alpha=.72$. To compute the variable we used scores' means.

\subsection{Quantitative analysis}

One of the goals of the current research was to assess whether pandemic influenced how people use technology - whether technology use increased or decreased in different use-contexts. To compare such changes, we used Wilcoxon ranked test. Our results indicate 


\begin{tabular}{|l|l|l|l|l|l|}
\hline & Work & Information & Entertainment & Health & Communication \\
\hline Parameter & & & & & \\
\hline Lealth Consciousness & -.23 & .12 & .01 & $.39^{*}$ & -.07 \\
Tracking & $-.46^{* *}$ & .08 & $.42^{* *}$ & -.15 & $.30^{*}$ \\
Concerns about pandemic & .218 & .04 & .09 & .18 & $.26^{*}$ \\
\hline$R^{2}$ & $.29^{* *}$ & .11 & -.07 & $.25^{*}$ \\
Adjusted $R^{2}$ & .11 & .07 & .06 & .05 & .10 \\
\hline Number of observations & .09 & .05 & .04 & .03 & .08 \\
\hline
\end{tabular}

Table 1: Beta coefficients and significance levels for multiple multivariate regression model. $\left[{ }^{*}\right] p<.05 ;\left[{ }^{* *}\right] p<.01$.

that the use of technology during the pandemic significantly differs, $p<.001$, compared to technology use before the pandemic. There was a significant increase in use of technology compared to pre-pandemic in the contexts technology used for: work, $z=-8.6$ (pre-pandemic $M=4.51$, during $M=5.16$ ); information gathering, $z=-9.2$ (pre-pandemic $M=4.53$, during $M=5.16$ ); entertainment $z=-8.2$ (pre-pandemic $M=4.41$, during $M=4.98$ ); communication $z=-12,3$ (pre-pandemic $M=3.92$, during $M=5.27$ ); and health, $z=-5.82$ (pre-pandemic $M=3.15$, during $M=3.4$ ). The largest number of participants reporting the increase was in the context of communication $(n=180)$ and work $(n=112)$. However, there were several participants reporting no change in the use of technology in each of the five contexts.

Another goal of this research was to examine factors that cause changes in the use of technology, depending on the context. In particular, we wanted to find to what extent the use of technology is influenced by health consciousness, the two dimensions of perceived isolation: loneliness, social support, concerns about pandemic tracking, and general concern about the pandemic. We first ran a correlation analysis. There was a positive significant correlation between use of technology for work and concerns about pandemic $(r=.20, p<.01)$, and pandemic tracking $(r=0.17, p<.05)$, and negative correlation with loneliness $(r=-.20, p<.01)$. Use of technology for information gathering was positively significantly correlated with concerns about the pandemic $(r=.25, p<.01)$, health consciousness $(r=.15, p<.05)$, and pandemic tracking $(r=.14, p<.05)$. Loneliness was positively significantly related to use of technology for entertainment $(r=.22, p<.01)$. Using technology for health significantly correlated with health consciousness $(r=.19, p<.01)$ and pandemic tracking $(r=.14, p<.05)$. Use of technology for communication significantly correlated with concerns about pandemic $(r=.23, p<.01)$, loneliness $(r=.16, p<.05)$, and pandemic tracking $(r=.23, p<.01)$. Concerns about pandemic significantly correlated with health consciousness $(r=.31, p<.01)$ and pandemic tracking $(r=.38, p<.01)$; and health consciousness with pandemic tracking $(r=.30, p<.01)$.

Considering these small to medium correlations, we ran multiple multivariate regressions. The dependent variables in the model included the five contexts of using the technology during-pandemic. The independent variables were all variables that significantly correlated with dependent variables. The results are presented in Table 1. Our findings imply that a low level of loneliness and a high level of concerns about the pandemic predict the increased use of technology for work. Similarly, the high concerns about the pandemic are a significant predictor of using technology for information gathering and education. According to our results, loneliness is a significant predictor of using technology for entertainment, and health consciousness of using technology for health. Lastly, our findings imply that the increased use of technology for communication results from high levels of loneliness, interest in pandemic tracking, and concerns about the pandemic.

\subsection{Qualitative analysis}

Our survey included an optional open-ended question inquiring about participants' comments they would like to add regarding their use of technology, behavior, and the pandemic. Out of the 218 participants only 72 provided valid input to the question. Responses were analyzed using thematic analysis, resulting in three categories: beneficial opportunities, concerns \& consequences, and behavioral transformations \& adaptations.

Twenty of the respondents highlighted only positive aspects in "beneficial opportunities", whereas 15 highlighted negative ones in "concerns \& consequences", and 4 mentioned both. The rest, 33, mainly mentioned "behavioral transformations \& adaptations" due to COVID-19.

4.2.1 Beneficial opportunities. Beneficial aspects included mentions of increased work productivity, efficiency, and providing future possibilities of working from home (12). Learning new ways and opportunities of using technologies was highlighted by 7 participants; one mentioned that they "become more confident" in using the technology, and another mentioned that "People are much more willing to engage with tech and try new things." Eight people mainly mentioned their appreciation of the opportunities provided by the technology in general during this time; one mentioned that it is a "life saver" during the lockdown, being in a risk group, 5 mentioned the significance of technology in providing means to communicate with family and friends specifically.

4.2.2 Concerns \& consequences. The effects of COVID-19 and technology have resulted in concerns and consequences. There were 7 mentions related to health concerns (anxiety, extended screen time, and physical health), and 2 mentions of loneliness. Four mentions of the pressure to work and be always available as a consequence of the technology. Whereas there were 5 mentions the negative effects that technology has on their work (losing their job), social life, internet quality, life quality (dominating life), and distance from nature. 
4.2.3 Behavioral transformations \& adaptations. Changes in behaviors relate to transformations and adaptations of technological use. There were 7 mentions of constant use of devices, acquisition of devices, or buying new technologies/software. Seeking news and information about the COVID-19 testing and/or analysis was mentioned by 7 respondents. There were also 7 mentions of the increase of technology use in the work context, whereas 4 related their reduced use of technology to their lack of work. The increase of using technology for entertainment content consumption and seeking communication with people was mentioned by 6 . Some comments (5) reflected the current situation, noting self-reflection, awareness of dependency on technology, dependency on online shopping, and possible over lasting effects of the current pandemic.

\section{DISCUSSION}

Our empirical investigation confirmed that the COVID-19 pandemic contributed to the increase of using technology by users in all contexts surveyed. The quantitative and qualitative results suggest that the increase of use has largely been in work and communications contexts, which could entail use out of necessity, i.e., to get work done. That could be the case for those who are working remotely and are concerned about the pandemic. However, it should be noted that not everyone had the possibility of working remotely, as many are unemployed or have lost their jobs due to COVID-19. Some participants highlighted positive aspects, appreciating the indirect effects of the pandemic, e.g., productivity, working remotely.

It is not surprising that people concerned with COVID-19 are indeed increasing their use of technology for information seeking and interest in pandemic tracking, as some participants mentioned news outlets and other sources to keep up to date. Health consciousness appears to significantly affect technology use for health context. It might be the case that individuals seek new ways to improve their lifestyles given the restrictions imposed on their physical activities. More health-conscious individuals might use more health-related applications, which corroborate with past research findings [5]. In the pandemic context, such applications might be targeting both physical and mental wellness to help mitigate the effects of the pandemic.

Furthermore, participants highlighted loneliness, in the open question, as a negative impact of COVID-19. A coping mechanism for the COVID-19 situation could be the use of technologies for entertainment $[22,30]$. Our quantitative results corroborate such assumption, showing that lonely individuals tend to use technologies for entertainment more.

\subsection{Practical implications}

Our exploratory findings suggest that crises, such as a global pandemic, increase technology use in different contexts. However, regression analyses show that different factors might be more or less significant considering this increase. Technology designers could use these findings when developing new technologies, helping people mitigate pandemic effects. For instance, it seems that people use entertainment and communication technologies to overcome loneliness. However, currently, most communication technologies rely on the fact that people already have contacts they could communicate with. This calls for research on communication technologies and innovative social entertainment applications that reach out to people who do not necessarily have a large social network, e.g., by suggesting connections with special interest groups or similar.

On the other hand, our findings imply the possibility that the increase in the use of communication technology might be a result of concerns about pandemic and tracking. This calls for better solutions that provide people with verified information because communicating with others to find out about the virus spread might increase misinformation and misconceptions related to the pandemic, e.g., inaccurate advice on preventive measures. The threat to an individual's well-being is also a concern with misinformation. It has been reported that people who are susceptible to misinformation are less inclined to follow health guidelines and to take vaccinations [34]. Especially in social media, rumors and misinformation have been on the rise during COVID-19 [39]. We believe that communication technologies should include the development of social media tools and applications to combat misinformation and provide guidelines from credible health resources.

Additionally, our results imply that pandemic concerns might increase the use of technology for work; however, a high level of loneliness might push people away from it. This might be interpreted as a signal that loneliness could potentially decrease work and productivity. Such interpretation implies that future research could consider developing work-assisted technologies that engage users equivalently to the social interactions present in the not-remote work environment. Similarly, it is also important to consider the long-term effects of loneliness and prolonged isolation on mental health. The importance of mental health technology has been stressed for the ongoing COVID-19 situation [13].

\subsection{Limitations and future work}

We note that our study has the following limitations. First, we conducted a self-reported, online survey and gathered participants with a snowball sampling, resulting in the relatively small sample size, making our findings less generalizable. Second, we ran the study in summer 2020, at an early stage of the COVID-19 pandemic. We intended to investigate the impact of COVID-19 early on during the pandemic and whether there were significant changes then. Third, only a small number of participants responded to the open-ended question, which might be due to respondents' fatigue. However, our study was short (average completion time 4.4 minutes). Hence, we believe that the low number of responses is due to the noncompulsory nature of the question. Consequently, the voluntary answers of our participants might be more reliable and honest.

We stress that this research purpose was exploratory and aimed to provide a basis for a further comparative study. Currently, we are in the process of completing the design of a more comprehensive study, drawing on our exploratory findings. Such work will enable us to identify whether the approach to using technology has changed, e.g., whether people refrained from excessive use of technology due to fatigue or developed a habit, or even addiction resulting in extensive reliance on technology.

\section{CONCLUSION}

We present results of an online survey exploring changes in the use of technology caused by the COVID-19 pandemic. The increase in 
the use of technology across all contexts investigated appear to be affected by both the pandemic and different intrinsic factors. We outline how our insights can be used to research and design applications supporting end-users' needs and consequentially improving their health and well-being.

\section{REFERENCES}

[1] Marshall H Becker, Lois A Maiman, John P Kirscht, Don P Haefner, and Robert H Drachman. 1977. The Health Belief Model and prediction of dietary compliance: a field experiment. Fournal of Health and Social Behavior 18, 4 (1977), 348-366.

[2] Ana I. Bento, Thuy Nguyen, Coady Wing, Felipe Lozano-Rojas, Yong-Yeol Ahn, and Kosali Simon. 2020. Information Seeking Responses to News of Local COVID19 Cases: Evidence from Internet Search Data. arXiv:2004.04591 [cs.SI]

[3] Alexander Bick, Adam Blandin, and Karel Mertens. 2020. Work from home after the COVID-19 Outbreak.

[4] Dale E Brashers, Daena J Goldsmith, and Elaine Hsieh. 2002. Information seeking and avoiding in health contexts. Human communication research 28, 2 (2002), 258-271.

[5] Miao Chao, Dini Xue, Tour Liu, Haibo Yang, and Brian J. Hall. 2020. Media use and acute psychological outcomes during COVID-19 outbreak in China. Fournal of Anxiety Disorders 74, April (2020), 102248. https://doi.org/10.1016/j.janxdis. 2020.102248

[6] A Chevance, D Gourion, N Hoertel, P-M Llorca, P Thomas, R Bocher, M-R Moro, V Laprévote, A Benyamina, P Fossati, et al. 2020. Ensuring mental health care during the SARS-CoV-2 epidemic in France: A narrative review. L'encephale 46 , 3 (2020), 193-201.

[7] Jaehee Cho, Dongjin Park, and H. Erin Lee. 2014. Cognitive factors of using health apps: Systematic analysis of relationships among health consciousness, health information orientation, eHealth literacy, and health app use efficacy. Fournal of Medical Internet Research 16, 5 (2014), 1-10. https://doi.org/10.2196/jmir.3283

[8] Erin York Cornwell and Linda J. Waite. 2009. Social Disconnectedness, Perceived Isolation, and Health among Older Adults. Fournal of Health and Social Behavior 50, 1 (2009), 31-48. https://doi.org/10.1177/002214650905000103 PMID: 19413133.

[9] Shivangi Dhawan. 2020. Online learning: A panacea in the time of COVID-19 crisis. Fournal of Educational Technology Systems 49, 1 (2020), 5-22.

[10] Mohan J Dutta-Bergman. 2004. Health attitudes, health cognitions, and health behaviors among Internet health information seekers: population-based survey. Journal of medical Internet research 6, 2 (2004), e15.

[11] Mohan J Dutta-Bergman. 2004. Primary sources of health information: Comparisons in the domain of health attitudes, health cognitions, and health behaviors. Health communication 16, 3 (2004), 273-288.

[12] Gunther Eysenbach. 2009. Infodemiology and infoveillance: framework for an emerging set of public health informatics methods to analyze search, communication and publication behavior on the Internet. Fournal of medical Internet research 11, 1 (2009), e11.

[13] Caroline A. Figueroa and Adrian Aguilera. 2020. The Need for a Mental Health Technology Revolution in the COVID-19 Pandemic. Frontiers in Psychiatry 11 (2020), 523. https://doi.org/10.3389/fpsyt.2020.00523

[14] Dana Rose Garfin. 2020. Technology as a coping tool during the coronavirus disease 2019 (COVID-19) pandemic: Implications and recommendations. Stress and Health 36 (10 2020), 555-559. Issue 4. https://doi.org/10.1002/smi.2975

[15] Joseph A Gliem and Rosemary R Gliem. 2003. Calculating, interpreting, and reporting Cronbach's alpha reliability coefficient for Likert-type scales.

[16] Mostafa Haghi, Kerstin Thurow, and Regina Stoll. 2017. Wearable devices in medical internet of things: scientific research and commercially available devices. Healthcare informatics research 23, 1 (2017), 4-15.

[17] Jonathan Haidt and Nick Allen. 2020. Digital technology under scrutiny. Nature 578, 7794 (2020), 226-227.

[18] Tzung Jeng Hwang, Kiran Rabheru, Carmelle Peisah, William Reichman, and Manabu Ikeda. 2020. Loneliness and social isolation during the COVID-19 pandemic. International Psychogeriatrics 32 (10 2020), 1217-1220. Issue 10 . https://doi.org/10.1017/S1041610220000988

[19] Rama K. Jayanti and Alvin C. Burns. 1998. The antecedents of preventive health care behavior: An empirical study. Fournal of the Academy of Marketing Science 26, 1 (1998), 6-15. https://doi.org/10.1177/0092070398261002

[20] Renate Klar and Dirk Lanzerath. 2020. The ethics of COVID-19 tracking appschallenges and voluntariness. Research Ethics 16, 3-4 (2020), 1-9.

[21] Amit Kramer and Karen Z Kramer. 2020. The potential impact of the Covid-19 pandemic on occupational status, work from home, and occupational mobility.

[22] Willy C Kriz. 2020. Gaming in the Time of COVID-19.

[23] Livio Luzi and Maria Grazia Radaelli. 2020. Influenza and obesity: its odd relationship and the lessons for COVID-19 pandemic. Acta diabetologica 57, 6 (2020), 759-764.

[24] Giorgio Marinoni, Hilligje Van't Land, and Trine Jensen. 2020. The impact of Covid-19 on higher education around the world.
[25] Amaryllis Mavragani. 2020. Tracking COVID-19 in Europe: infodemiology approach. $7 M I R$ public health and surveillance 6, 2 (2020), e18941.

[26] Long Chiau Ming, Noorazrina Untong, Nur Amalina Aliudin, Norliza Osili, Nurolaini Kifli, Ching Siang Tan, Khang Wen Goh, Pit Wei Ng, Yaser Mohammed Al-Worafi, Kah Seng Lee, et al. 2020. Mobile health apps on COVID-19 launched in the early days of the pandemic: content analysis and review. FMIR mHealth and uHealth 8, 9 (2020), e19796.

[27] Marco Narici, Giuseppe De Vito, Martino Franchi, Antonio Paoli, Tatiana Moro, Giuseppe Marcolin, Bruno Grassi, Giovanni Baldassarre, Lucrezia Zuccarelli, Gianni Biolo, Filippo Giorgio di Girolamo, Nicola Fiotti, Flemming Dela, Paul Greenhaff, and Constantinos Maganaris. 2020. Impact of sedentarism due to the COVID-19 home confinement on neuromuscular, cardiovascular and metabolic health: Physiological and pathophysiological implications and recommendations for physical and nutritional countermeasures. European fournal of Sport Science 0, 0 (2020), 1-22. https://doi.org/10.1080/17461391.2020.1761076 PMID: 32394816.

[28] National Cancer Institute. 2017. Health Information National Trends Survey CYCLE 2 | HINTS-FDA. https://hints.cancer.gov/view-questions-topics/questiondetails.aspx?red=1\&qid=757. (Accessed on 01/09/2021).

[29] Frederick H Navarro and Stephen T Wilkins. 2001. A new perspective on consumer health Web use:" valuegraphic" profiles of health information seekers. Managed care quarterly 9, 2 (2001), 35-43.

[30] Amber Pahayahay and Najmeh Khalili-Mahani. 2020. What Media Helps, What Media Hurts: A Mixed Methods Survey Study of Coping with COVID-19 Using the Media Repertoire Framework and the Appraisal Theory of Stress. Fournal of medical Internet research 22, 8 (2020), e20186.

[31] Tiago Peçanha, Karla Fabiana Goessler, Hamilton Roschel, and Bruno Gualano. 2020. Social isolation during the COVID-19 pandemic can increase physical inactivity and the global burden of cardiovascular disease. American fournal of Physiology-Heart and Circulatory Physiology 318, 6 (2020), H1441-H1446.

[32] Public Health Agency of Sweden. 2021. Regulations and general guidelines - The Public Health Agency of Sweden. https://www.folkhalsomyndigheten.se/thepublic-health-agency-of-sweden/communicable-disease-control/covid19/regulations- and-general-guidelines/. (Accessed on 02/24/2021).

[33] RFE/RL Radio Free Europe/Radio Liberty. 2020. COVID-19: Armenian, Moldovan, Romanian Police Enforce National Lockdowns. https://www.rferl.org/a/covid19-coronavirus-roundup-march-25-confirmed-cases-crisis/30508199.html. (Accessed on $02 / 24 / 2021$ ).

[34] Jon Roozenbeek, Claudia R Schneider, Sarah Dryhurst, John Kerr, Alexandra LJ Freeman, Gabriel Recchia, Anne Marthe Van Der Bles, and Sander Van Der Linden. 2020. Susceptibility to misinformation about COVID-19 around the world. Royal Society open science 7, 10 (2020), 201199.

[35] Leia Y. Saltzman, Tonya Cross Hansel, and Patrick S. Bordnick. 2020. Loneliness, Isolation, and Social Support Factors in Post-COVID-19 Mental Health. Psychological Trauma: Theory, Research, Practice, and Policy 12 (2020), S55-S57. Issue S1. https://doi.org/10.1037/tra0000703

[36] Samantha L Schneider and Martha Laurin Council. 2020. Distance learning in the era of COVID-19. Archives of Dermatological Research 8 (2020), 1.

[37] Syed Ghulam Sarwar Shah, David Nogueras, Hugo Cornelis van Woerden, and Vasiliki Kiparoglou. 2020. The COVID-19 Pandemic: A Pandemic of Lockdown Loneliness and the Role of Digital Technology. Fournal of Medical Internet Research 22, 11 (2020), e22287.

[38] Barbara G Tabachnick, Linda S Fidell, and Jodie B Ullman. 2007. Using multivariate statistics. Vol. 5. Pearson, Boston, MA.

[39] Samia Tasnim, Md Mahbub Hossain, and Hoimonty Mazumder. 2020. Impact of rumors and misinformation on COVID-19 in social media. Fournal of preventive medicine and public health 53, 3 (2020), 171-174.

[40] Andrew Urbaczewski and Young Jin Lee. 2020. Information Technology and the pandemic: a preliminary multinational analysis of the impact of mobile tracking technology on the COVID-19 contagion control. European fournal of Information Systems 29, 4 (2020), 405-414.

[41] Jessica Vitak and Michael Zimmer. 2020. <? covid19?> More Than Just Privacy: Using Contextual Integrity to Evaluate the Long-Term Risks from COVID-19 Surveillance Technologies. Social media+ society 6, 3 (2020), 2056305120948250.

[42] Herbert Wanga, Thobius Joseph, and Mauna Belius Chuma. 2020. Social Distancing: Role of Smartphone During Coronavirus (COVID-19) Pandemic Era. International fournal of Computer Science and Mobile Computing 9, 5 (2020), 181-188.

[43] World Health Organization (WHO). 2021. WHO Coronavirus Disease (COVID-19) Dashboard | WHO Coronavirus Disease (COVID-19) Dashboard. https://covid19. who.int/. (Accessed on 01/03/2021).

[44] World Health Organization (WHO). NA. Constitution. https://www.who.int/ about/who-we-are/constitution. (Accessed on 01/08/2021)

[45] World Health Organization (WHO). NA. Naming the coronavirus disease (COVID19) and the virus that causes it. https://www.who.int/emergencies/diseases/novelcoronavirus-2019/technical-guidance/naming-the-coronavirus-disease-(covid2019)-and-the-virus-that-causes-it. (Accessed on 01/11/2021).

[46] World Health Organization (WHO). 2021. Weekly operational update on COVID-19 - 5 January 2021. https://www.who.int/publications/m/item/weekly- 
epidemiological-update---5-january-2021. (Accessed on 01/11/2021).

[47] Worldometer. 2021. COVID-19 CORONAVIRUS PANDEMIC-Worldometer. https //www.worldometers.info/coronavirus/. (Accessed on 01/05/2021).

[48] Yanxiang Yang and Joerg Koenigstorfer. 2020. Determinants of physical activity maintenance during the Covid-19 pandemic: a focus on fitness apps. Translational behavioral medicine 10, 4 (2020), 835-842.

[49] Martin Zens, Arne Brammertz, Juliane Herpich, Norbert Südkamp, and Martin Hinterseer. 2020. App-based tracking of self-reported COVID-19 symptoms: analysis of questionnaire data. Journal of medical Internet research 22, 9 (2020), e21956.

\section{APPENDIX}

(1) Health Consciousness

Participants instructions: Please, indicate to what extent do you agree with the following statements [scores from 1 strongly disagree to 5 - strongly agree]

- I worry that there are harmful chemicals in my food.

- I am concerned about my drinking water quality.

- I usually read the ingredients on food labels.

- I read more health-related articles than I did 3 years ago.

- I am interested in information about my health. I am concerned about my health all the time.

(2) Perceived isolation: Loneliness.

Participants instructions: How often do you... [scores: 1 hardly ever, 2 - some of the time, 3 - often]

- Feel that you lack companionship?

- Feel left out?

- Feel isolated from others?

(3) Perceived isolation: Social support.

Participants instructions: How often do you... [scores: 1 - often, 2 - some of the time, 3 - hardly ever (or never), 4 - I have none]

- Open up to members of your family?

- Rely on members of your family?

- Open up to your friends?
- Rely on your friends?

- Open up to your spouse or partner?

- Rely on your spouse or partner?

(4) Use of technology before/after corona.

Participants instructions: Before/After corona pandemic...

[scores before: 1-Never, 5 - Every day; after: 1 - Much less, 5 - Much more]

- I used/use technologies for work

- I used/use technologies for information gathering and education (news, online videos,courses..etc..)

- I used/use technologies for entertainment (games, streaming tv, music..etc.)

- I used/use technologies for health (fitness apps, videos, etc.)

- I used/use technologies for communication (social networks, chats, video calls)

(5) Pandemic tracking and concern.

Participants instructions: To what extent do you agree with the following sentences [scores: 1 - strongly disagree, 5 strongly agree]

- I would like to have access to information that shows those who are infected with coronavirus around me.

- If I were infected with coronavirus, I would share that information so that others know I am infected and can take precaution.

- I think that tracking of people infected with coronavirus is needed.

- I am concerned with the coronavirus pandemic.

(6) Open-ended question.

Participants instructions: Is there anything you would like to add about the corona virus and its influence on your technology use or on your behavior? 